\title{
Comparing Lateral Modulation and Amplitude Modulation in Phantom Sensation
}

\author{
Tao Morisaki ${ }^{\left({ }^{\otimes}\right)}$ (D), Masahiro Fujiwara(D), Yasutoshi Makino®D, \\ and Hiroyuki Shinoda(D
}

The University of Tokyo, 5-1-5 Kashiwanoha, Kashiwa-shi, Chiba-ken 277-8561, Japan

morisaki@hapis.k.u-tokyo.ac.jp, Masahiro_Fujiwara@ipc.i.u-tokyo.ac.jp, \{yasutoshi_makino,hiroyuki_shinoda\}@k.u-tokyo.ac.jp

\begin{abstract}
Phantom Sensation (PhS) is a tactile illusion in which a single sensation is elicited by stimulating two distant points. That sensation moves continuously between the two stimuli by changing the amplitude ratio. In this paper, we compared $\mathrm{PhS}$ for two types of tactile stimuli: lateral modulation (LM) of $20 \mathrm{~Hz}$ and amplitude modulation (AM) of $200 \mathrm{~Hz}$. In LM, a stimulus point is moved periodically and laterally by several millimeters. In AM, the pressure is changed periodically at a fixed stimulation point. LM and AM are produced by ultrasound radiation pressure, where the force intensity of LM and AM were changed with the same temporal pattern. The results showed that the continuity and the localization of $\mathrm{PhS}$ elicited by $\mathrm{LM}$ at $20 \mathrm{~Hz}$ were significantly smaller than those elicited by AM at $200 \mathrm{~Hz}$ in 18 out of 24 conditions. However, $\mathrm{PhS}$ remained in all conditions that we used, regardless of LM or AM, even for an extremely long duration of $7.5 \mathrm{~s}$ and a short duration of $0.5 \mathrm{~s}$.
\end{abstract}

Keywords: Phantom sensation $\cdot$ Tactile receptor $\cdot$ Ultrasound.

\section{Introduction}

When stimulating two distant points on the human skin, a single sensation is felt between them. This phenomenon is called Phantom Sensation (PhS). In $\mathrm{PhS}$, a continuous tactile motion is presented by changing the amplitude ratio between the distant stimulation points [7]. The illusion is useful to minimize the number of stimulators that simplify the tactile display device [1,9]; however, the mechanism and properties are not well clarified.

In this study, we examined the conditions to elicit $\mathrm{PhS}$ using ultrasound radiation pressure that can control the stimulus quantitatively with high reproducibility and controllability. In particular, we compared $\mathrm{PhS}$ elicited by lateral

This work was supported in part by JSPS Grant-in-Aid for Scientific Research (S) 16H06303 and JST CREST JPMJCR18A2.

(C) The Author(s) 2020

I. Nisky et al. (Eds.): EuroHaptics 2020, LNCS 12272, pp. 122-130, 2020.

https://doi.org/10.1007/978-3-030-58147-3_14 
modulation (LM) [6] and amplitude modulation (AM) [4] presented by ultrasound. LM is the stimulus in which an ultrasound focus is moved periodically and laterally by several millimeters. AM is the stimulus in which the pressure amplitude of an ultrasound focus is modulated periodically at a fixed point. The details of LM and AM are described in Sect.2.2. LM is clearly perceived even below $50 \mathrm{~Hz}$, and AM is strongly perceived above $100 \mathrm{~Hz}$. As the authors' subjective view, LM is perceived to be small and localized near the stimulus point, and $\mathrm{AM}$ is perceived as more widespread.

In the experimental design, we considered that low-frequency LM and highfrequency AM are typical stimuli that selectively stimulate type-I and FAII mechanoreceptors, respectively, where type-I includes fast-adapting (FAI) and slowly adapting (SAI) mechanoreceptors and FAII indicates fast-adapting type-II mechanoreceptors. The selectivity has not been evaluated quantitatively. However, we tentatively assumed it because it is plausible from the temporal characteristics of the threshold and the perceived spatial area. In the following experiments, we compared the difference in the perceived tactile motion continuity and localization of PhS elicited by LM and AM, changing the intensity of LM and AM equally with the same temporal pattern.

Some studies have been conducted on $\mathrm{PhS}$ using non-vibratory stimuli as well as various vibrotactile stimuli at $100 \mathrm{~Hz}$ and $30 \mathrm{~Hz}[1,2]$. However, there is no comparison between the different types of stimuli under the same conditions. In this paper, we carefully measured the pressure pattern on the skin, and equalized the temporal profile of the stimulus between LM and AM.

\section{Principle}

\subsection{Phantom Sensation}

In our experiment, we simultaneously stimulated two points on the palm to elicit PhS by airborne ultrasound phased array (AUPA) [5]. AUPA creates an ultrasound focus (stimulus point) of approximately $1 \mathrm{~cm}^{2}$. The maximum force of AUPA we used is approximately $4.8 \mathrm{~g}$, as shown in Fig. 5 (right) and higher than the perception threshold on the hand [8]. AUPA is described in Appendix.

Figure 1 (C) shows a schematic illustration of the $\mathrm{PhS}$ evaluated in this paper. The presented pressure was changed linearly and logarithmically by 5 and 6 described in Appendix. Linear and logarithmic changes have been widely used in $\mathrm{PhS}$ experiments $[1,9,10]$. In Fig. $1, L_{p}$ is the length between two stimulated points, and $\boldsymbol{u}_{p}$ is the moving direction vector of $\operatorname{PhS}\left(\left\|\boldsymbol{u}_{p}\right\|=1\right) . P_{1}^{\text {Linear }}$ and $P_{2}^{\text {Linear }}$ are the pressures presented at $\boldsymbol{r}_{\mathrm{f} 1}$ and $\boldsymbol{r}_{\mathrm{f} 2}$, respectively, when changing the pressure linearly. When changing the pressure logarithmically, the corresponding pressure is defined as $P_{1}^{\log }$ and $P_{2}^{\log } . P_{k}^{\text {Linear }}$ and $P_{k}^{\log }(k=1,2)$, are shown in Fig. 1 ( $\mathrm{A}$ and $\mathrm{B}$ ) and given by

$$
\begin{aligned}
P_{k}^{\text {Linear }}(t) & =P_{k}^{\max }\left(2-k+\frac{t}{d_{p}}\right), \\
P_{k}^{\log }(t) & =P_{k}^{\max } \log _{2}\left[3-k+(-1)^{k} \frac{t}{d_{p}}\right],
\end{aligned}
$$


(A)

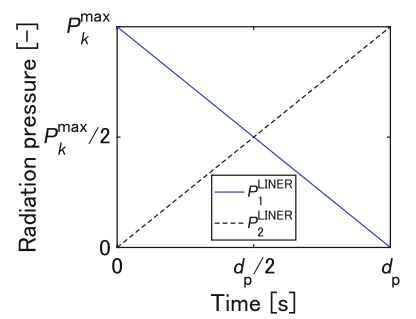

(C)

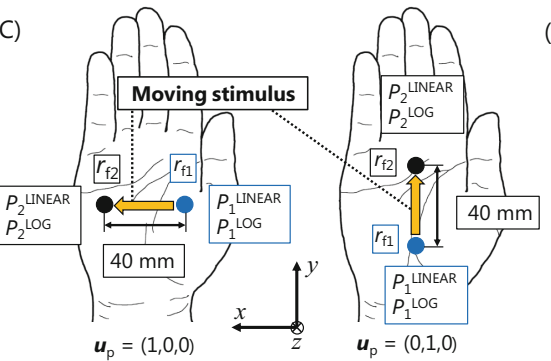

(B)

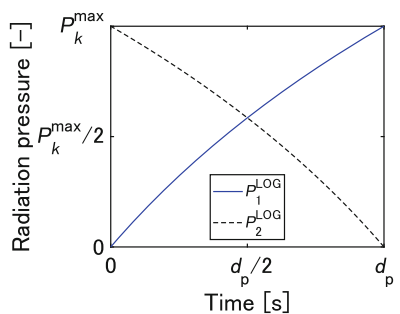

(D)

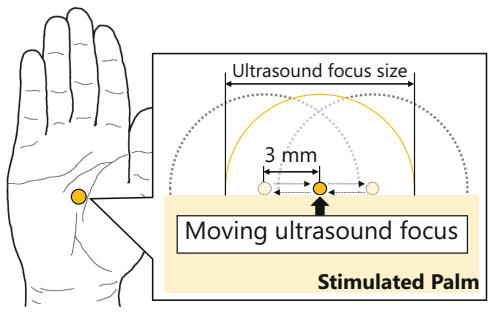

Fig. 1. A) \& B) Changes in pressure linearly and logarithmically $\left(P_{k}^{\text {Linear }}\right.$ and $\left.P_{k}^{\log }\right)$. C) Schematic illustration of Phantom Sensation evaluated in this paper. D) Schematic illustration of lateral modulation.

where $d_{p}$ is the duration of $\mathrm{PhS}$ and $P_{k}^{\max }$ is the maximum pressure that AUPAs can present. The range of $t$ is $0 \leq t \leq d_{p}$.

\subsection{Lateral Modulation and Amplitude Modulation}

Amplitude modulation: AM is the method to present a vibrotactile stimulus by periodically modulating the pressure amplitude of an ultrasound focus [4]. Previous research showed that the sensitivity of RAII is highest at approximately $200 \mathrm{~Hz}$ and higher than that of RAI and SAI at the same frequency [3]. Therefore, $\mathrm{AM}$ at $200 \mathrm{~Hz}$ is considered to stimulate RAII more strongly than RAI and SAI.

Lateral modulation: LM is the ultrasound stimulus method that moves an ultrasound focus periodically and laterally several millimeters while maintaining the intensity constant [6] (Fig. 1-D). LM at $20 \mathrm{~Hz}$ is more strongly perceived than AM at the same frequency [6]. Moreover, at $20 \mathrm{~Hz}$, the sensitivity of RAII is lower than that of RAI and SAI [3]. Therefore, we used LM at $20 \mathrm{~Hz}$ to stimulates RAI and SAI more strongly than RAII.

\section{Experimental Methods}

In this experiment, we compared PhS elicited by LM and AM in terms of continuity and localization. Continuity and localization are typical elements of $\mathrm{PhS}$ that have been evaluated in many previous studies $[1,2,9,10]$. 

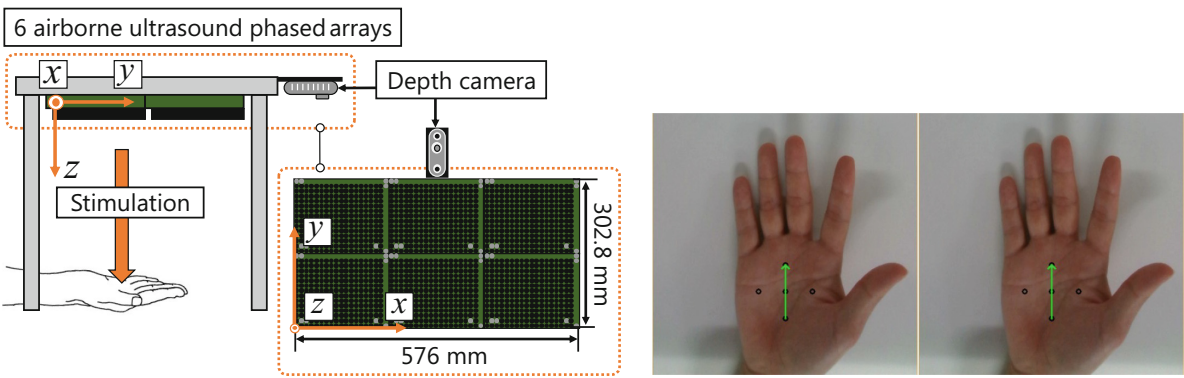

Fig. 2. Left: Experimental setup presenting Phantom Sensation. Right: Video of participants' hand captured by a depth camera with markers.

Stimulus: The experimental setup is shown in Fig. 2 (left). The setup consists of 6 AUPAs and a depth camera (Intel Real Sense D435). The coordinate system is a right-handed system with an origin at the lower left of AUPAs. We presented two ultrasound foci to $\boldsymbol{r}_{\mathrm{f} 1}=\left(308,151.4, z_{h 1}\right) \mathrm{mm}$ and $\boldsymbol{r}_{\mathrm{f} 2}=\left(268,151.4, z_{h 2}\right) \mathrm{mm}$, then $\boldsymbol{u}_{p}=(1,0,0)$. We also presented the foci to $\boldsymbol{r}_{\mathrm{f} 1}=\left(288,131.4, z_{h 1}\right) \mathrm{mm}$ and $\boldsymbol{r}_{\mathrm{f} 2}=\left(288,171.4, z_{h 2}\right) \mathrm{mm}$, then $\boldsymbol{u}_{p}=(0,1,0)$. $z_{h 1}$ and $z_{h 2}$ are the heights of the participants' hand measured by the depth camera. In $\mathrm{PhS}$, we used $L_{p}=40 \mathrm{~mm}, d_{p}=1.5,2.5,4.0 \mathrm{~s}$, and a linear and logarithmic function shown in Fig. 1 (A and B) as the amplitude change. The stimulus type was LM and AM stimuli. The pressure of AM at a $200 \mathrm{~Hz}$ sinusoidal wave, where the perception threshold of RAII is less than $-10 \mathrm{~dB}[3]$. In LM, the ultrasound focus was moved laterally at a constant speed and periodically at $20 \mathrm{~Hz}$. The movement width was $6 \mathrm{~mm}$. The motion direction of LM was perpendicular to $\boldsymbol{u}_{p}$. AM and LM had the same maximum pressure. As a whole, we varied the stimulus type (LM or AM), the type of amplitude change (linear or logarithm), $d_{p}$, and $\boldsymbol{u}_{p}$. Therefore, there were $2 \times 2 \times 3 \times 2=24$ trials for each set. Each participant performed three sets. All trials were performed in the same order, which was randomized once.

Procedure: 10 male participants (ages $23-27$ ) were included in the experiment. Participants put their hands toward the radiation surface of AUPAs. Participants saw the video of the hand captured by the depth camera. The video is shown in Fig. 2 (right). Participants adjusted the position of their hand so that the center of the circle-shaped markers added to the video was aligned with the center of their hand. The direction of $\mathrm{PhS}$ was also indicated by a green arrow in the video. After adjustment, $\mathrm{PhS}$ was presented, and participants answered the following two questions using a 7-grade Likert scale (1-7): Q. 1) The presented stimulus is continuously moving stimulation, $Q$. 2) The presented stimulus consisted of only one stimulus point. We intended that Q. 1 and Q. 2 evaluated the continuity and the localization of $\mathrm{PhS}$, respectively. After answering the questions, the participants readjusted the position of their hands and proceeded to the next trial. 
A) Q. 1 Continuity
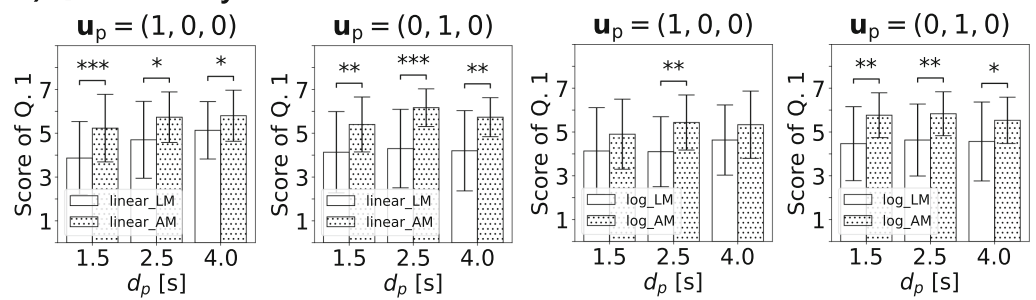

\section{A) Q. 2 Localization}
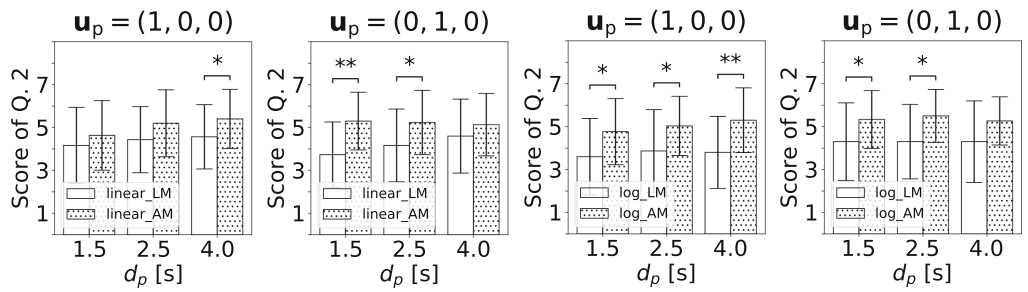

\section{B) Q. 1 Continuity}

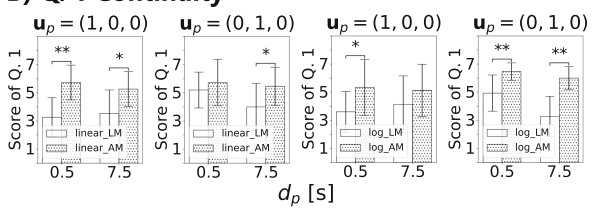

B) Q. 2 Localization

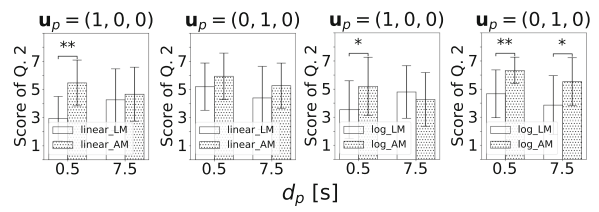

Fig. 3. Average scores of Q. 1 (continuity) and Q. 2 (localization). Error bars mean standard deviation. A) Stimulus duration $d_{p}=1.5,2.5,4.0 \mathrm{~s}$. B) $d_{p}=0.5,7.5 \mathrm{~s}$.

\subsection{Result}

The average scores of Q. 1 (continuity) and Q. 2 (localization) are shown in Fig. 3 (A). The AM score was higher than the LM score in all conditions. Wilcoxon signed-rank test showed that the differences between the AM scores and the LM scores were significant in the Q. 1-linear case $(p<0.05)$. In the Q. 2-logarithm case, the differences were significant when $\left\{\boldsymbol{u}_{p}=(1,0,0)\right.$ and $\left.d_{p}=2.5 \mathrm{~s}\right\}$, and $\left\{\boldsymbol{u}_{p}=(0,1,0)\right.$ and $\left.d_{p}=1.5,2.5,4.0 \mathrm{~s}\right\}$. In the Q. 2-linear case, the differences were significant when $\left\{\boldsymbol{u}_{p}=(1,0,0)\right.$ and $\left.d_{p}=4.0 \mathrm{~s}\right\}$, and $\left\{\boldsymbol{u}_{p}=(0,1,0)\right.$ and $\left.d_{p}=1.5,2.5 \mathrm{~s}\right\}$. The significant differences with $p<0.05, p<0.005$, and $p<$ 0.0005 were indicated by $* * *$, and $* * *$, respectively, in Fig. 3 .

We applied ANOVA to the results with the fore factors: the type of stimulus, the type of amplitude change, $\boldsymbol{u}_{p}$, and $d_{p}$. We observed that the type of stimulus $\left(F(1,714)=173.3640, p=6.6093 \times 10^{-17}<0.0001\right)$ and $d_{p}(F(2,714)=$ 4.9319, $\left.p=7.4604 \times 10^{-3}<0.05\right)$ had significant effects on the scores of $\mathrm{Q}$. 1. We also observed that only the type of stimulus $(F(1,714)=106.2125, p=$ $\left.2.5904 \times 10^{-23}<0.0001\right)$ had significant effects on the scores of Q. 2 . 

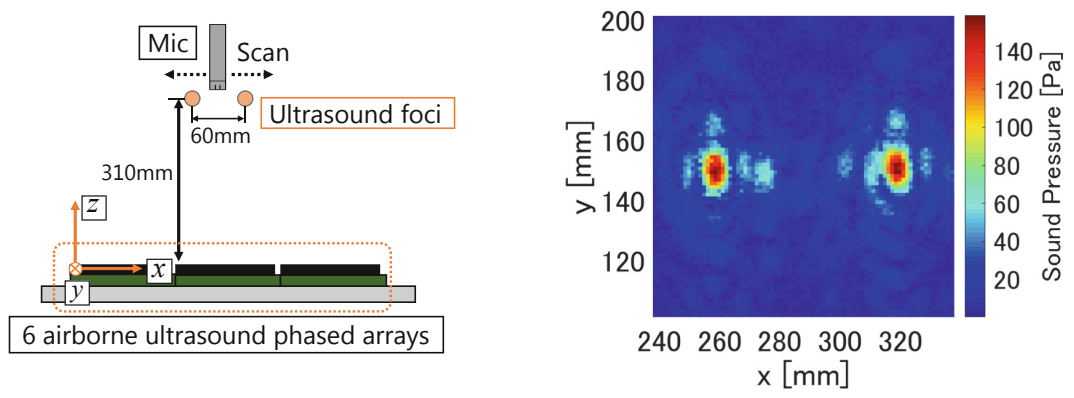

Fig. 4. Left: Experimental setup. Right: Measured sound distribution of the two ultrasound foci.
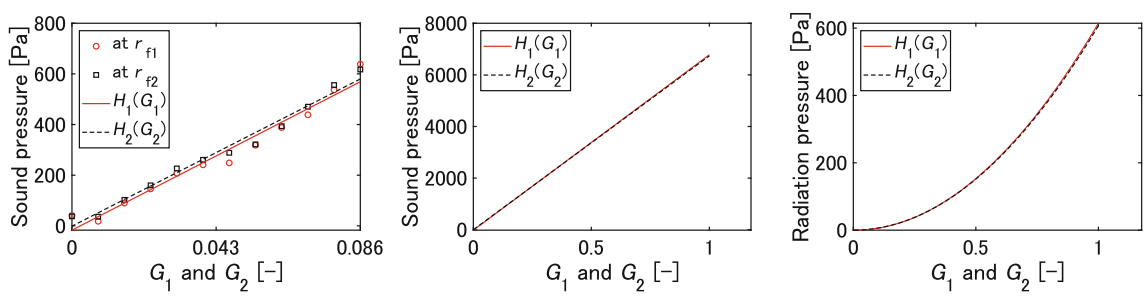

Fig. 5. Left: Measured change in sound pressure, according to $G_{1}$ and $G_{2}$. The results of linear regression $H_{1}$ and $H_{2}$ were also added. Center \& Right: Calculated sound pressure and radiation pressure in $0 \leq G_{1} \leq 1,0 \leq G_{2} \leq 1$.

\subsection{Discussion}

Originally, we expected that LM cannot elicit $\mathrm{PhS}$ because LM is perceived to be localized near the stimulus point. The results showed that AM elicited PhS more easily than LM, but $\mathrm{PhS}$ remained in the LM conditions. Therefore, we compared LM and AM again with extremely long or short durations of $\mathrm{PhS}$ $\left(d_{p}=0.5,7.5 \mathrm{~s}\right)$, where eliciting $\mathrm{PhS}$ may be difficult. Our results and previous studies showed that the stimulus duration $d_{p}$ has significant effects on $\mathrm{PhS}$ [10].

However, contrary to the authors' expectations, LM and AM still elicited PhS under extreme conditions. Moreover, the localization of LM was greater than that of AM in $\boldsymbol{u}_{p}=(1,0,0), d_{p}=7.5 \mathrm{~s}$. The results are shown in Fig 3 (B). 5 males (ages $24-26$ ) participated in the second experiment. The experimental procedure is the same as that described in Sect. 3 .

These results indicated that $\mathrm{AM}$ tends to elicit PhS more easily than LM, but both $\mathrm{AM}$ and $\mathrm{LM}$ can elicit $\mathrm{PhS}$ even under the extreme conditions. Note that there are differences in the stimulus area and perception intensity between LM and AM. The stimulus area of LM was larger than that of AM because of the movement of the stimulus points. The perceived intensities of LM and AM differ for the same driving power [6]. In this experiment, we did not equalize the perceived intensities but maximized it in both cases to make the participants clearly perceive the stimuli. These differences should be explored in future work. 


\section{Conclusion}

In this paper, we compared Phantom Sensation $(\mathrm{PhS})$ elicited by lateral modulation (LM) of $20 \mathrm{~Hz}$ and amplitude modulation (AM) of $200 \mathrm{~Hz}$ in terms of continuity and localization. This comparison aims to clarify the conditions for eliciting PhS. LM and AM were produced by ultrasound with high reproducibility and controllability. The same temporal pattern was applied to the force intensities of LM and AM.

The results showed that the continuity and the localization of $\mathrm{PhS}$ elicited by LM were significantly smaller than those elicited by AM in 18 out of 24 conditions. However, Phs remained in all conditions we used, regardless of LM or AM, even when the stimulus duration of $\mathrm{PhS}$ having significant effects on PhS [10] was extremely long or short (7.5 s or $0.5, \mathrm{~s})$.

\section{Appendix: Airborne Ultrasound Phased Array}

In our experiment, the ultrasound stimulus was produced by an airborne ultrasound phased array (AUPA) [5]. A single unit of AUPA that we used was provided with 249 ultrasound transducers. AUPA presents a non-contact force with about $1 \mathrm{~cm}^{2}$ circle area. The pressure is called acoustic radiation pressure. In this section, we measured the sound pressure by AUPA to change the intensity of LM and AM with the same temporal pattern (Eq. 1, 2).

Presenting $\mathrm{PhS}$ requires two-point stimuli [7]. When AUPA creates a focus at $\boldsymbol{r}_{\mathrm{f} 1}$ and $\boldsymbol{r}_{\mathrm{f} 2}$ simultaneously, the sound pressure $p_{\mathrm{f}}$ at $\boldsymbol{r}$ and $t$ is as follows:

$$
\begin{aligned}
p_{\mathrm{f}}\left(t, \boldsymbol{r}, \boldsymbol{r}_{\mathrm{f} 1}, \boldsymbol{r}_{\mathrm{f} 2}\right) & =\sum_{i=1}^{N_{\mathrm{trans}}} p_{i}(t, \boldsymbol{r})\left(G_{1} \mathrm{e}^{-\mathrm{j}\left\|\boldsymbol{r}_{\mathrm{f} 1}-\boldsymbol{r}_{i}\right\|}+G_{2} \mathrm{e}^{-\mathrm{j}\left\|\boldsymbol{r}_{\mathrm{f} 2}-\boldsymbol{r}_{i}\right\|}\right), \\
p_{i}(t, \boldsymbol{r}) & =\frac{A D\left(\theta_{i}\right)}{\left\|\boldsymbol{r}-\boldsymbol{r}_{i}\right\|} \mathrm{e}^{-\beta\left\|\boldsymbol{r}-\boldsymbol{r}_{i}\right\|} \mathrm{e}^{\mathrm{j}\left(\nu\left\|\boldsymbol{r}-\boldsymbol{r}_{i}\right\|-\omega t\right)},
\end{aligned}
$$

where $\boldsymbol{r}_{i}$ is the position of each transducer, $N_{\text {trans }}$ is the total number of transducers, $A$ is the maximum amplitude of a transducer, $D$ is the directivity of a transducer, $\omega$ is the frequency of ultrasound, $\nu$ is the wavenumber of the ultrasound we used, $\beta$ is the attenuation coefficient of air, $\mathrm{j}$ is the imaginary unit, and $\theta_{i}$ is the angle from the transducer centerline to a focus. $G_{1}$ and $G_{2}$ are coefficients to determine the amplitude of two foci, respectively; thus, $0 \leq G_{1}, 0 \leq G_{2}, G_{1}+G_{2} \leq 1$.

We measured the sound distribution of two foci created by 6 AUPAs at $\boldsymbol{r}_{\mathrm{f} 1}=(258,151.4,310) \mathrm{mm}$ and $\boldsymbol{r}_{\mathrm{f} 2}=(318,151.4,310) \mathrm{mm}$ using Eq. 3. The experimental setup is shown in Fig. 4 (left). The sound distribution was measured by a microphone (Brüel \& Kjær Type 2670) moved by a three-axis stage in $100 \mathrm{~mm} \times 100 \mathrm{~mm}(238 \leq x \leq 338,101.4 \leq y \leq 201.4, z=310 \mathrm{~mm})$ by $1.5 \mathrm{~mm}$. The AUPAs output was $6.2 \%$ of the maximum value $\left(G_{1}=0.031\right.$ and $G_{2}=$ 0.031 ) because the maximum sound pressure which the microphone can measure is about $316 \mathrm{~Pa}$. The measured sound distribution is shown in Fig. 4 (right). 
To change the radiation pressure accurately and obtain $P_{k}^{\max }$ of 6 AUPAs, we measured the change in sound pressure according to $\boldsymbol{G}=\left(G_{1}, G_{2}\right)$. The experimental setup is the same as that shown in Fig. 4 (left). 6 AUPAs created two foci at $\boldsymbol{r}_{\mathrm{f} 1}=(258,151.4,310) \mathrm{mm}$ and $\boldsymbol{r}_{\mathrm{f} 2}=(318,151.4,310) \mathrm{mm}$. We changed $\boldsymbol{G}$ from $(0.086,0)$ to $(0,0.086)$ by $(-0.0078,0.0078)$ and measured the sound pressure at $\boldsymbol{r}_{\mathrm{f} 1}$ and $\boldsymbol{r}_{\mathrm{f} 2}$ in each case. The measured sound pressure and the result of linear regression are shown in Fig. 5 (left). $H_{1}$ and $H_{2}$, which are sound pressure according to $\boldsymbol{G}$ at $\boldsymbol{r}_{\mathrm{f} 1}$ and $\boldsymbol{r}_{\mathrm{f} 2}$, respectively, are as follows:

$$
\begin{aligned}
& H_{1}\left(G_{1}\right)=1.7323 \times 10^{6} G_{1}-17.0363, \\
& H_{2}\left(G_{2}\right)=1.7182 \times 10^{6} G_{2}-1.3803 .
\end{aligned}
$$

The sound pressure and calculated radiation pressure [5] in $0 \leq G_{1} \leq 1,0 \leq$ $G_{2} \leq 1$ are shown in Fig. 5 (center and right).

\section{References}

1. Alles, D.S.: Information transmission by phantom sensations. IEEE Trans. Man Mach. Syst. 11(1), 85-91 (1970)

2. Culbertson, H., Nunez, C.M., Israr, A., Lau, F., Abnousi, F., Okamura, A.M.: A social haptic device to create continuous lateral motion using sequential normal indentation. In: 2018 IEEE Haptics Symposium (HAPTICS), pp. 32-39. IEEE (2018)

3. Gescheider, A., Bolanowski, S.J., Hardick, K.R.: The frequency selectivity of information-processing channels in the tactile sensory system. Somatosens. Mot. Res. 18(3), 191-201 (2001)

4. Hasegawa, K., Shinoda, H.: Aerial vibrotactile display based on multiunit ultrasound phased array. IEEE Trans. Haptics 11(3), 367-377 (2018)

5. Hoshi, T., Takahashi, M., Iwamoto, T., Shinoda, H.: Noncontact tactile display based on radiation pressure of airborne ultrasound. IEEE Trans. Haptics 3(3), 155-165 (2010)

6. Takahashi, R., Hasegawa, K., Shinoda, H.: Tactile stimulation by repetitive lateral movement of midair ultrasound focus. IEEE Transactions on Haptics 13, 334-342 (2019)

7. Von Békésy, G.: Neural funneling along the skin and between the inner and outer hair cells of the cochlea. J. Acoust. Soc. Am. 31(9), 1236-1249 (1959)

8. Weinstein, S.: Intensive and extensive aspects of tactile sensitivity as a function of body part, sex and laterality. The skin senses (1968)

9. Yatani, K., Truong, K.N.: Semfeel: a user interface with semantic tactile feedback for mobile touch-screen devices. In: Proceedings of the 22nd Annual ACM Symposium on User Interface Software and Technology, pp. 111-120. ACM (2009)

10. Yun, G., Oh, S., Choi, S.: Seamless phantom sensation moving across a wide range of body. In: 2019 IEEE World Haptics Conference (WHC), pp. 616-621. IEEE (2019) 
Open Access This chapter is licensed under the terms of the Creative Commons Attribution 4.0 International License (http://creativecommons.org/licenses/by/4.0/), which permits use, sharing, adaptation, distribution and reproduction in any medium or format, as long as you give appropriate credit to the original author(s) and the source, provide a link to the Creative Commons license and indicate if changes were made.

The images or other third party material in this chapter are included in the chapter's Creative Commons license, unless indicated otherwise in a credit line to the material. If material is not included in the chapter's Creative Commons license and your intended use is not permitted by statutory regulation or exceeds the permitted use, you will need to obtain permission directly from the copyright holder. 